\title{
BMJ Open Wordless intervention for people with epilepsy and learning disabilities (WIELD): a randomised controlled feasibility trial
}

\author{
Silvana E Mengoni, ${ }^{1}$ Bob Gates, ${ }^{2}$ Georgina Parkes, ${ }^{3}$ David Wellsted, ${ }^{1}$ \\ Garry Barton, ${ }^{4}$ Howard Ring, ${ }^{5}$ Mary Ellen Khoo, ${ }^{6}$ Deela Monji-Patel, ${ }^{6,7}$ \\ Karin Friedli, ${ }^{8}$ Asif Zia, ${ }^{3}$ Lisa Irvine, ${ }^{4}$ Marie-Anne Durand ${ }^{1,9}$
}

To cite: Mengoni SE, Gates B, Parkes G, et al. Wordless intervention for people with epilepsy and learning disabilities (WIELD): a randomised controlled feasibility trial. BMJ Open 2016;6: 012993. doi:10.1136/bmjopen-2016012993

- Prepublication history and additional material is available. To view please visit the journal (http://dx.doi.org/ 10.1136/bmjopen-2016012993).

Received 9 June 2016 Revised 1 August 2016 Accepted 25 August 2016

CrossMark

For numbered affiliations see end of article.

Correspondence to Dr Silvana E Mengoni; s.mengoni@herts.ac.uk

\section{ABSTRACT}

Objective: To investigate the feasibility of a full-scale randomised controlled trial of a picture booklet to improve quality of life for people with epilepsy and learning disabilities.

Trial design: A randomised controlled feasibility trial. Randomisation was not blinded and was conducted using a centralised secure database and a blocked 1:1 allocation ratio.

Setting: Epilepsy clinics in 1 English National Health Service (NHS) Trust.

Participants: Patients with learning disabilities and epilepsy who had: a seizure within the past 12 months, meaningful communication and a carer with sufficient proficiency in English.

Intervention: Participants in the intervention group used a picture booklet with a trained researcher, and a carer present. These participants kept the booklet, and were asked to use it at least twice more over 20 weeks. The control group received treatment as usual, and were provided with a booklet at the end of the study.

Outcome measures: 7 feasibility criteria were used relating to recruitment, data collection, attrition, potential effect on epilepsy-related quality of life (Epilepsy and Learning Disabilities Quality of Life Scale, ELDQOL) at 4-week, 12-week and 20-week follow-ups, feasibility of methodology, acceptability of the intervention and potential to calculate costeffectiveness.

Outcome: The recruitment rate of eligible patients was $34 \%$ and the target of 40 participants was reached. There was minimal missing data and attrition. An intention-to-treat analysis was performed; data from the outcome measures suggest a benefit from the intervention on the ELDQOL behaviour and mood subscales at 4 and 20 weeks follow-up. The booklet and study methods were positively received, and no adverse events were reported. There was a positive indication of the potential for a cost-effectiveness analysis.

Conclusions: All feasibility criteria were fully or partially met, therefore confirming feasibility of a definitive trial.

Trial registration number: ISRCTN80067039.

\section{Strengths and limitations of this study}

- This feasibility study is the first completed randomised controlled trial addressing the unmet information and self-management needs of people with learning disabilities and epilepsy.

- Effective collaboration with local clinical services and patient and public representatives facilitated recruitment to target.

- A mixed-methods design clearly addressed the feasibility criteria and highlighted improvements for a definitive trial through quantitative and qualitative data.

- Despite efforts to recruit people with severe learning disabilities, this population are underrepresented in the study.

- There were important variations in the extent that carers involved participants in the questionnaires, and how the booklet was used, which cannot be examined in a feasibility study.

\section{INTRODUCTION}

People with learning disabilities ( 1.5 million people in the UK) face an increased risk of health problems, poorer outcomes than in the general population and premature, avoidable deaths. ${ }^{1-3}$ Epilepsy is the most common neurological condition for people with learning disabilities ${ }^{4}$ with a prevalence rate of $22 \%,{ }^{5}$ compared with $0.4-1 \%$ of the general population, which increases with the severity of the learning disability. ${ }^{6}$

Epilepsy in people with learning disabilities typically begins in childhood, and has a complex presentation, with people often experiencing frequent seizures, generalised seizures and multiple seizure types. ${ }^{7}$ Poorly controlled and severe seizures are more likely than in the general population with $\sim 70 \%$ of people with learning disabilities 
and epilepsy continuing to have seizures despite taking antiepileptic medication. ${ }^{7} 8$ Comorbid health and mental health issues as well as cognitive impairment can make the management of epilepsy difficult. ${ }^{9}$ This can have a negative impact on care needs, daily activities, work, quality of life, instances of hospitalisation, mortality and health and social care costs. ${ }^{10-15}$

Guidance from the National Institute for Health and Care Excellence (NICE) in England states that people with learning disabilities and epilepsy should be offered the same services, investigations and care as the general population, as well as information and support that is tailored to their needs in order to promote autonomy and empowerment. ${ }^{16}$ However, this group continue to experience poor access to diagnostic investigations and specialist services; access to services with appropriate expertise appears to be highly dependent on location. 7151718

People with learning disabilities are often excluded from clinical trials ${ }^{19}$ resulting in a weak evidence base for effective treatment and services for this population. This is particularly evident in epilepsy research, ${ }^{12}$ illustrated by a recent review of service responses for epilepsy in learning disabilities which identified no evaluations using randomisation or a matched comparison group. ${ }^{15}$ Despite calls for non-pharmacological interventions to be used alongside antiepileptic medication, ${ }^{20}$ there is little evidence for the effectiveness of such interventions due to the lack of robust trials in this area. ${ }^{21}$

People with learning disabilities and epilepsy often lack appropriate skills training to manage their epilepsy, as well as provision of accessible information. ${ }^{12}{ }^{18}$ There is also a need to improve communication between healthcare professionals and people with learning disabilities regarding decision-making about epilepsy management. ${ }^{12}$ In the general population, self-management interventions have been found to improve people's understanding and management of epilepsy along with their tolerability and adherence to medication. ${ }^{22-24}$ In contrast, self-management interventions are rarely implemented or evaluated with people with learning disabilities and epilepsy. ${ }^{15}$ Such interventions have potential to improve knowledge and epilepsy management, with a likely improvement in quality of life.

'Getting on With Epilepsy' is a picture booklet designed for people with learning disabilities and epilepsy, ${ }^{26}$ and is part of the Beyond Words series of booklets. ${ }^{27}$ This booklet tells the story of a young adult with epilepsy, and promotes understanding of epilepsy, selfmanagement and empowerment. The booklet uses pictures rather than words to tell a story. It is suitable for people with a range of communication styles and capitalises on the visual literacy and pictorial superiority of people with learning disabilities, who tend to process and assimilate pictorial information better than textual information. ${ }^{28-30}$ The range of Beyond Words booklets are popular and have received awards, but have not, as yet, been evaluated in a controlled study, nor routinely adopted in the health or social care sector in the UK. The epilepsy picture booklet has the potential to be an inexpensive and simple way of addressing the information and self-management needs of people with learning disabilities and epilepsy.

The objectives of the Wordless Intervention for Epilepsy in Learning Disabilities (WIELD) randomised controlled feasibility trial (feasibility RCT) were to:

1. Assess the feasibility of undertaking a RCT of the picture booklet as an intervention for epilepsy in people with learning disabilities.

2. Explore the feasibility of collecting resource use and quality of life data so as to inform the design of the health economics component of a future definitive trial.

3. Assess the study procedures' and intervention's acceptability among adults with learning disabilities, carers and health professionals.

The full protocol for this study has previously been published. ${ }^{31}$

\section{METHODS}

\section{Design and setting}

The study had a two-arm, single-centre parallel design, and was conducted at one National Health Service (NHS) Trust in England where participants were Hertfordshire Partnership University NHS Foundation Trust (HPFT) patients over a 20-month period from July 2014 to February 2016. Participants were recruited through seven epilepsy clinics, and then randomised to the intervention or control group.

\section{Participants}

Eligible patients were 18 years of age or over, with a confirmed clinical diagnosis of a learning disability ( $\mathrm{IQ} \leq 70)$ and epilepsy. Patients had to have experienced at least one seizure over the previous 12 months and have meaningful verbal or non-verbal communication which was operationalised as being able to tell or follow the story in the picture booklet. Originally, only patients with verbal communication were eligible. This was changed before the start of recruitment based on feedback from patient representatives, carers and health professionals. The carers of the patients had to be sufficiently proficient in English to read and complete the study questionnaires. Patients were excluded if they had a visual impairment or dementia, or if they had used the picture booklet within the past 12 months.

A subgroup of participants and their carers were invited to take part in a semistructured interview after completion of the final follow-up assessment to explore feasibility and acceptability of the study and intervention. Random sampling was originally proposed, but this was modified to purposeful sampling to ensure a range of views and backgrounds were represented. A sample of health professionals were also invited to participate in a semistructured interview. 


\section{Consent}

Eligible patients were identified by epilepsy nurses and consultant psychiatrists who ran epilepsy clinics at HPFT. A $\log$ of eligible patients was created using the inclusion and exclusion criteria. These patients were then contacted to invite them to take part in the study through a postal invitation pack containing standard and easy-read information (see online supplementary material for easy-read information sheet). This was then followed up with a phone call $\sim 1$ week later. A researcher subsequently visited interested patients and carers to discuss the study. If the patient had capacity to decide whether to participate, they completed an easy-read consent form. If the carer felt that the patient did not have capacity, a consultee declaration form was used. Originally the visit was stated to occur at an epilepsy clinic but this was changed, before recruitment started, to allow patients and carers to choose to be seen at their home if that was convenient. The original proposal also stated that consenting, randomisation, data collection and the intervention would all be conducted by a researcher with a research nurse background. In order to maximise recruitment, this was changed during the study to allow a trained clinical studies officer to also take on this role.

\section{Randomisation and blinding}

Participants were randomly allocated individually online by a researcher using a database on a secure website. The randomisation (1:1 allocation ratio) was 'blocked' into groups of six. Owing to the nature of the intervention, it was not possible for the participants or research team to be blind to group allocation.

\section{Intervention}

The researchers who conducted the intervention received training from Beyond Words about how to use the booklet. Participants in the intervention condition, along with their carer, met the researcher at a convenient location. During this meeting, the 'Getting on With Epilepsy' booklet was introduced. This involved the participant going through the booklet at their own pace and being encouraged to talk about what they could see and how they felt, as appropriate.

As well as verbal guidance on how to use the booklet, the participant and carer were advised that there was further guidance and resources at the back of the booklet, and they were given further information produced by Beyond Words. Participants kept the booklet, and were asked to look at the booklet at least twice more at home over the 20-week study period. Participants or carers received a phone call $\sim 2$ weeks later to discuss any issues they may have had with the booklet. The duration of all contact between researcher, participant and carer was noted by the research team, in order to estimate the cost of the intervention.

Participants in the control condition received routine information and services. At the end of the study, control group participants were provided with a copy of the booklet and information about how to use it.

\section{Outcome measures}

The feasibility criteria were developed a priori to reflect key aspects of the study which would be necessary for a full-scale trial. The seven feasibility criteria were:

- Rate of eligible patients recruited is higher than $60 \%$.

- At least $80 \%$ of study measures have been completed.

- Discontinuation rates fall under $20 \%$.

- The likely location of the improvement in the primary outcome (quality of life) is at least $10 \%$.

- Good feasibility and acceptability of the study methodology.

- Good acceptability of the intervention among participants, carers and health professionals.

- Collection of resource use and quality of life data is feasible, and the increase in treatment cost will be minimal.

Each participant was assessed at baseline (T0), and at 4 (T1), 12 (T2) and 20 (T3) weeks after randomisation (figure 1) using the outcome measures detailed in table 1 . The primary outcome measure was the Epilepsy and Learning Disabilities Quality of Life Scale (ELDQOL).$^{32}{ }^{33}$ Seizure severity and control were secondary outcomes, as improved management of epilepsy has the potential to reduce seizures. Information was also collected about how often the intervention group participants used the booklet, how long for and who with. Participants were asked about any other epilepsy resources they had used, to explore whether booklet use led to further information-seeking.

The study questionnaires were designed to be completed by the carer with the participant's input, where possible. The baseline questionnaire was completed with a researcher present. The follow-up questionnaires were sent and returned by post.

The semistructured interview schedule for the health professionals and participants and carers aimed to explore the acceptability of the study design and intervention and perceived feasibility of using the booklet in a routine care setting. The interview schedule also explored the self-management, information and support needs of participants, in relation to their experience of epilepsy, and this is reported elsewhere. ${ }^{35}$

\section{Sample size}

One of the study objectives was to assess variation in the ELDQOL in order to determine whether the intervention is likely to achieve a $10 \%$ increase in the participants' quality of life and to allow exclusion of an effect size of $<0 .{ }^{36}$ Ten per cent equates to an effect size of $\sim 0.4$. In a full-scale trial, this would require a total sample size of $174(1-\beta=0.8, \alpha=0.05)$, which indicates a sample size of 16 per group (eg, 9\% per group) in the feasibility study. Assuming a conservative $25 \%$ drop out, the recruitment target was a sample size of 20 per group (40 participants in total). For the qualitative aspect of 


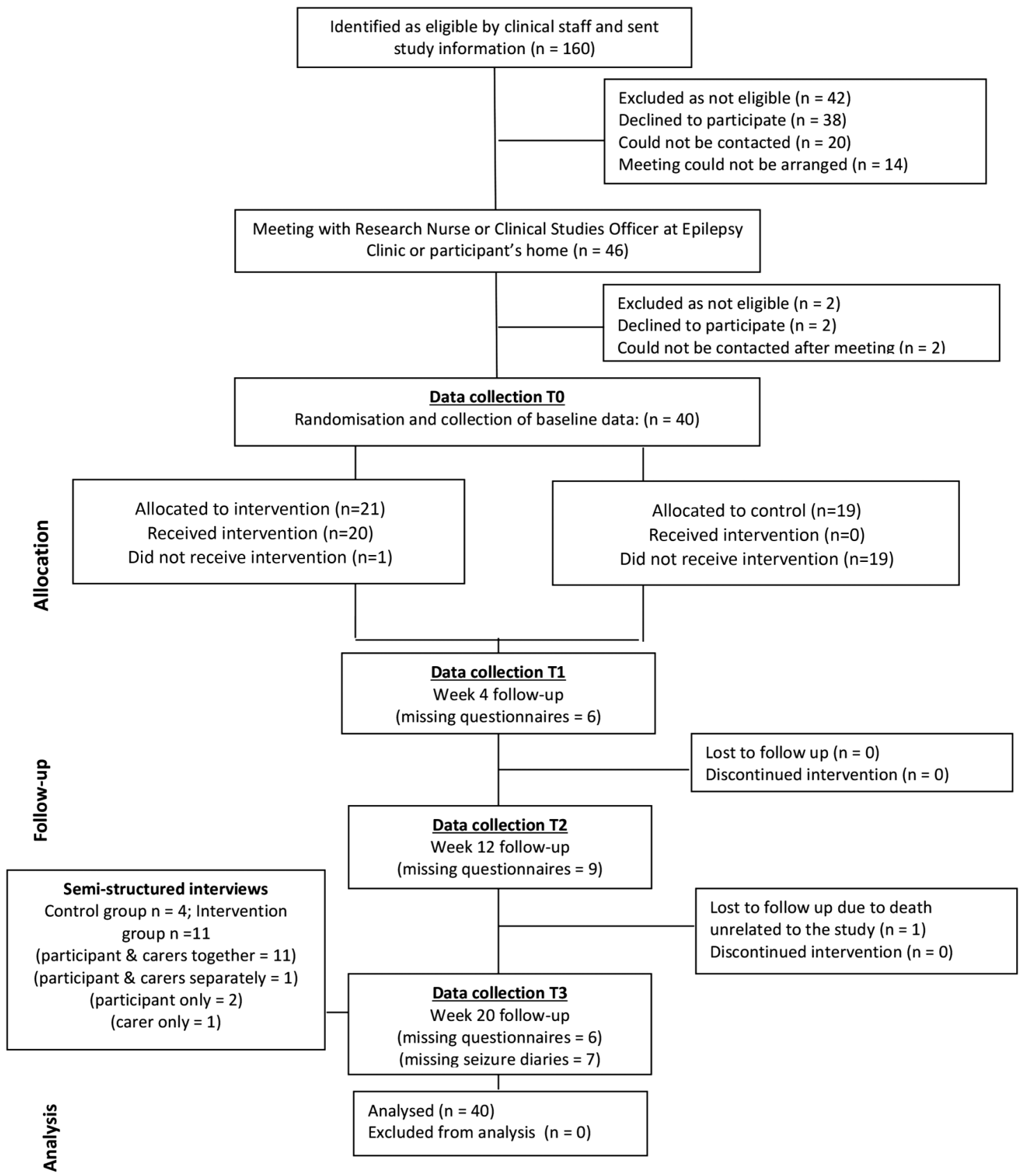

Figure 1 Study flow chart.

the study, we aimed to interview a subgroup of 15 participants and carers.

\section{Data analysis}

The analysis aimed to determine the feasibility of conducting a full-scale RCT by addressing the objectives and feasibility criteria outlined in table 2.

Quantitative data analysis was undertaken on Stata V.13 (StataCorp. Stata Statistical Software: Release 13. College Station, Texas: StataCorp LP; 2013). Most analysis was descriptive to evaluate the flow of patients through the study, the proportion of missing questionnaires, demographics of participants, use of the picture booklet and seizure control. For the primary outcome,
ELDQOL, the variability of the scores for the four subscales were estimated at each follow-up time point in line with Cocks and Torgerson. ${ }^{36}$ Cohen's d effect sizes for the difference between the two groups were calculated. The $80 \%$ CIs of the effect sizes were examined to determine whether they excluded an effect size of zero, that is, an indication of a difference between the two groups, and included the target effect size, that is, a $10 \%$ advantage for the intervention group.

For the health economic analysis, levels of resource use associated with the booklet, intervention staff training, face-to-face meetings (including travel), and the 2-week follow-up call were recorded, and unit costs (£GBP for the 2013-2014 financial year) were subsequently assigned 
Table 1 Study outcome measures

Outcome

Data collection method $\begin{array}{llll}\text { T0 } & \text { T1 } & \text { T2 } & \text { T3 } \\ \text { Baseline } & \text { Week 4 } & \text { Week 12 } & \text { Week 20 }\end{array}$ $\begin{array}{llll}\text { T0 } & \text { T1 } & \text { T2 } & \text { T3 } \\ \text { Baseline } & \text { Week 4 } & \text { Week 12 } & \text { Week 20 }\end{array}$

Number of eligible patients and

number recruited

Discontinuation rates across both

groups and reasons

Demographic data

\section{Case report form}

Case report form

Questionnaire: context-specific questions about age, sex, ethnicity, and living circumstances of the participant, the type of care provided by the carer and their relationship to the participant

Patterns of use of the Beyond Words booklet (intervention group only) Use of other epilepsy-related information

Quality of life as the primary outcome measure

Questionnaire: context-specific questions

Questionnaire: context-specific questions

Questionnaire: ELDQOL scale, which consists of four subscales: behaviour, seizure severity, mood and side effects $^{32} 33$

Seizure severity as a secondary outcome measure

Questionnaire: ELDQOL seizure severity subscale

Seizure control as a secondary

outcome measure

Health-related quality of life

Seizure diary

Questionnaire: EQ-5D-5L index and visual analogue scale (EQ-VAS) - proxy version $^{34}$

Health and social services and resources use

Feasibility and acceptability of the study procedures

Use and perceived usefulness of existing resources and services Information and self-management support needs

Perceived acceptability of the intervention

Perceived barriers and facilitators to Semistructured interview $\checkmark$ Questionnaire: context-specific resource use questions

Semistructured interview

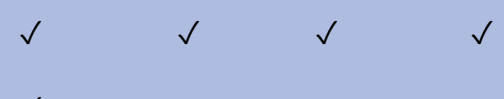

Semistructured interview

Semistructured interview

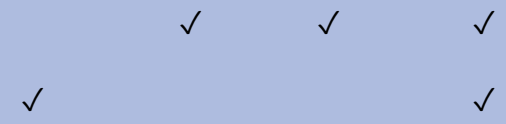

the use and dissemination of the

intervention in routine care

Semistructured interview
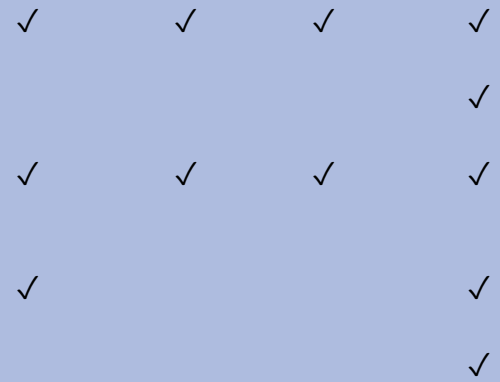

to all items. ${ }^{37-40}$ The total costs were then apportioned across all participants in the intervention arm, in order to estimate mean per participant cost of the intervention. In order to estimate overall cost to the NHS, Personal Social Services (PSS), and informal care, the carer was asked to complete a resource use questionnaire at baseline and 20-week follow-up.

Health-related quality of life was estimated using the EuroQol EQ-5D-5L questionnaire, ${ }^{34}$ a generic preference-based measure. EQ-5D-5L data were converted into utility scores (a scale where zero is equal to death and one is full health) ${ }^{41}$ using a mapping approach based on the three-level version. ${ }^{42}$ Quality-adjusted life year (QALY) scores were subsequently calculated using the area under the curve approach. ${ }^{43}$ As this was a feasibility study, the main focus was on completion rates.
A preliminary within-trial analysis (using bivariate regression, ${ }^{44}$ based on a complete-case analysis ${ }^{45}$ ) was also conducted over the 20-week follow-up period, though these results need to be treated with caution due to small numbers. The incremental cost-effectiveness ratio (ICER), defined as mean incremental cost/mean incremental effect, was subsequently estimated. ${ }^{41}$ NICE generally deems interventions which have an ICER below $£ 20000$ per QALY as cost-effective. ${ }^{46}$

The semistructured interviews were transcribed and analysed using thematic content analysis ${ }^{47}$ on NVivo V.11. Two members of the research team coded the interviews, with $30 \%$ of the interviews being coded by both researchers. Codes were compared and then discussed, and themes were derived through discussion. 


\begin{tabular}{ll}
\hline Objectives & Feasibility criteria (if applicable) \\
\hline Evaluate the recruitment rate and number & Feasibility criterion 1: rate of eligible
\end{tabular}

Evaluate the recruitment rate and number of eligible patients recruited (quantitative)

Evaluate completion rates (quantitative)

Measure discontinuation rates across both arms (quantitative)

Assess the variability of the primary

outcome measure (quality of life)

(quantitative)

Assess the feasibility and acceptability of the study methodology (qualitative)

Explore potential weaknesses of the study design (qualitative) patients recruited is higher than $60 \%$

Feasibility criterion met

and suggested

amendments if not met

Criterion not met overall

(although target recruitment

was met)

Amendments:

- Extend recruitment period

- Develop materials to increase recruitment of patients with severe

learning disabilities

Feasibility criterion 2 : at least $80 \%$ of Criterion met study measures have been

completed

Feasibility criterion 3 : discontinuation rates fall under $20 \%$

Feasibility criterion 4 : the likely location of the improvement in the primary outcome (quality of life) is at least $10 \%$.

Feasibility criterion 5 : good feasibility and acceptability of the study methodology

\section{Criterion met}

Criterion partially met

Amendments:

- Omit medication subscale

- Develop an epilepsy knowledge measure

\section{Criterion met}

- Target recruitment was met $(n=40)$

- Per cent of patients recruited from all those screened: $25 \%$

- Per cent of patients recruited from eligible screened patients: $34 \%$

- $85 \%$ of all study measures were completed.

No discontinuations with the exception of one death (unrelated to the study)

- The $80 \% \mathrm{Cl}$ of the effect size excludes zero in favour of the intervention group on ELDQOL behaviour (T1; T3) and mood subscales (T1; T3)

- The $80 \% \mathrm{Cl}$ of the effect size includes the target effect size (10\% improvement) for ELDQOL behaviour (T3).

- Qualitative data indicated high acceptability and feasibility of the study methods.

Suggestions for improvements have been identified, for example,

1. Reduce and alter questionnaire material

- Remove 12-week follow-up assessment as there was most missing data $(23 \%)$

- Remove medication side-effects subscale of ELDQOL

Develop and add epilepsy knowledge measure

2. Provide several completion formats for follow-up questionnaires

- Option to complete an online questionnaire or answer questions over the phone

3. Minimise missing data at 20 weeks

- 20-week questionnaire will be completed during a study visit with the research nurse

4. Understand how booklet is used by participants and carers 
Table 2 Continued

Feasibility criterion met

and suggested

Objectives

Feasibility criteria (if applicable)

Explore the acceptability of the Beyond

Words booklet (qualitative)

To explore the feasibility of collecting resource use and quality of life data (quantitative)
Feasibility criterion 6: good acceptability of the intervention among participants, carers and health professionals

Feasibility criterion 7 : collection of resource use and quality of life data is feasible, and the increase in treatment cost will be minimal.

Monitor the intervention's patterns of use postrandomisation, and explore whether other resources on epilepsy have been used (quantitative)

amendments if not met

Criterion met
Criterion partially met amendments:

- Costs may be lower in a full-scale trial as the training costs are apportioned per participant.

\section{Outcome}

- Conduct observations of booklet use at 20-week follow-up

- High levels of acceptability of the booklet in intervention group

- Use of booklet perceived to be feasible in routine care

- Good rates of data completion and minimal data cleaning was required.

- The mean per participant cost of the intervention was £122.

- Preliminary cost-effectiveness analysis indicates no significant difference between the two groups.

- $86 \%$ of participants used the booklet at least once more at home.

- The number of participants in the intervention group $(n=21)$ reporting to have used the intervention on the follow-up questionnaires decreased over time from 15 (4 weeks) to 12 (12 weeks) to 10 (20 weeks)

- Minimal use of other education/information resources

ELDQOL, Epilepsy and Learning Disabilities Quality of Life Scale. 


\section{RESULTS}

Four out of seven criteria used to determine the feasibility of a full-scale trial were fully met (table 2). Three criteria were partially met. Amendments to the study design and methodology have been identified and would be implemented in a definitive trial. The participant flow diagram for the study can be seen in figure 1 .

\section{Recruitment}

Recruitment began in July 2014 and was originally scheduled to be completed by December 2014. The recruitment period was extended by 4 months to compensate for logistical issues that caused delays at the study onset. The target of 40 participants was met in April 2015, without delaying the planned study completion date. All participants and carers chose to be visited at home by the researcher. Follow-up data collection continued until September 2015, and this is when participants' involvement in the study ended.

The first feasibility criterion was to randomise $60 \%$ of eligible patients. The study recruited $25 \%$ of all screened patients $(n=160)$ and $34 \%$ of screened patients who were eligible $(n=118)$. Despite not meeting this feasibility criterion, the study recruited to target without delay to the study end date.

The most common reasons for being excluded from the trial was the carer reporting that the patient would be unable to use the booklet due to communication issues or an absence of seizures within the past 12 months. There was a range of reasons for people declining to take part, although approximately half of the carers/patients did not give a reason.

The characteristics of participants across the two groups can be seen in table 3 . There are some differences between the groups. In particular, the intervention group had had their epilepsy diagnosis for fewer years. They were also slightly younger and received fewer hours of care from their carer.

\section{Data collection}

The proportion of completed study measures was $85 \%$ with most missing data at T2 $(23 \%)$, thus meeting the second feasibility criterion overall (at least $80 \%$ of study measures completed). The proportion of missing data was similar across the two groups. Two participants did not complete any follow-up questionnaires (T1-T3), but did not ask to withdraw from the study (despite the opportunity to do so).

\section{Discontinuation rates}

No participants withdrew from the trial. One participant died over the course of the study, unrelated to study participation. One participant was considered not to have received the intervention as they refused to look at the booklet with the research nurse and with their carer at a later date. An intention-to-treat analysis was carried out, including these participants. Therefore, the third feasibility criterion (discontinuation rates under $20 \%$ ) was met.

\begin{tabular}{|c|c|c|}
\hline & $\begin{array}{l}\text { Control group } \\
(n=19)\end{array}$ & $\begin{array}{l}\text { Intervention } \\
\text { group }(n=21)\end{array}$ \\
\hline \multicolumn{3}{|l|}{ Gender } \\
\hline Female:male & $10: 9$ & $13: 8$ \\
\hline \multicolumn{3}{|l|}{ Age } \\
\hline Mean (SD) & $44.68(14.53)$ & 39.00 (12.65) \\
\hline \multicolumn{3}{|l|}{ Ethnicity } \\
\hline White & 19 & 19 \\
\hline Bangladeshi & 0 & 1 \\
\hline Black一other & 0 & 1 \\
\hline \multicolumn{3}{|l|}{ Years since diagnosis* } \\
\hline Mean (SD) & $39.31(16.22)$ & 23.38 (16.49) \\
\hline \multicolumn{3}{|l|}{ Accommodation } \\
\hline Family home & 6 & 3 \\
\hline Own property & 0 & 1 \\
\hline $\begin{array}{l}\text { Registered care } \\
\text { home }\end{array}$ & 5 & 7 \\
\hline $\begin{array}{l}\text { Supported } \\
\text { accommodation }\end{array}$ & 8 & 9 \\
\hline Tenancy & 0 & 1 \\
\hline \multicolumn{3}{|c|}{ Carer relationship to participant } \\
\hline $\begin{array}{l}\text { Family member: paid } \\
\text { carer }\end{array}$ & $6: 13$ & $5: 16$ \\
\hline \multicolumn{3}{|c|}{ Number of hours of care per week provided by carer } \\
\hline Median (IQR) & $37(35-168)$ & $15(10-168)$ \\
\hline
\end{tabular}

\section{Intervention effect on primary and secondary outcomes}

The intervention group generally reported higher scores at baseline (T0) compared with the control group on the ELDQOL, indicating poorer quality of life. Therefore, adjusted means for the follow-up time points were calculated controlling for baseline differences. Effect sizes were then calculated for the group difference for the outcome measures, along with $80 \%$ CIs. Table 4 shows the baseline mean scores and SDs, adjusted T1-T3 mean scores and CIs, along with effect sizes and CIs. Examination of effect sizes and CIs indicated a signal of a positive effect from the intervention on the behaviour and mood subscales of the ELDQOL at T1 and T3. On subscales for behaviour, mood and seizure severity (secondary outcome) at T2, there was indication of improved scores in the control group. This finding is unexpected and there is no theoretical explanation; the most likely reason for this finding is the relatively small sample size at T2 due to the largest proportion of missing data being at this time point (23\%).

Participants reported difficulties completing the ELDQOL medication subscale. Respondents were often unable to determine whether a behaviour was caused by epilepsy medication (and could therefore be identified as a side effect of epilepsy medication), or was due to other medication or other reasons. Furthermore, the seizure subscale was only completed for participants who had experienced a seizure over the previous 4 weeks. The sample size was often very low: $8-11$ people in the 
Table 4 ELDQOL mean scores, SDs and 95\% Cls with T1-T3 adjusted for baseline

\begin{tabular}{|c|c|c|c|c|c|c|c|c|c|}
\hline & \multirow[t]{2}{*}{$\mathbf{N}$} & \multicolumn{3}{|c|}{ Control group } & \multirow[t]{2}{*}{$\mathbf{N}$} & \multicolumn{3}{|c|}{ Intervention group } & \multirow{2}{*}{$\begin{array}{l}\text { Effect size of the group } \\
\text { difference }(80 \% \mathrm{Cl})\end{array}$} \\
\hline & & Mean & SD & $95 \%$ Cls & & Mean & SD & $95 \% \mathrm{Cls}$ & \\
\hline \multicolumn{10}{|c|}{ ELDQOL seizure severity } \\
\hline T0 (baseline) & 12 & 27.50 & 8.29 & - & 11 & 29.25 & 9.37 & - & - \\
\hline T1 (4 weeks) & 10 & 26.42 & - & 22.46 to 30.39 & 8 & 26.66 & - & 22.50 to 30.82 & $0.04^{*}(-0.17$ to 0.25$)$ \\
\hline T2 (12 weeks) & 11 & 24.26 & - & 20.27 to 28.25 & 9 & 29.17 & - & 25.01 to 33.33 & $0.79^{*}(0.43$ to -1.15$)$ \\
\hline T3 (20 weeks) & 13 & 25.62 & - & 21.68 to 29.56 & 8 & 27.06 & - & 22.68 to 31.44 & $0.21^{*}(-0.02$ to 0.44$)$ \\
\hline \multicolumn{10}{|c|}{ ELDQOL side effects } \\
\hline T0 (baseline) & 11 & 71.56 & 4.93 & - & 15 & 65.99 & 10.75 & - & - \\
\hline T1 (4 weeks) & 12 & 65.66 & - & 50.02 to 72.29 & 17 & 65.98 & - & 60.84 to 71.12 & $0.03^{*}(-0.13$ to 0.19$)$ \\
\hline T2 (12 weeks) & 13 & 66.57 & - & 60.10 to 73.05 & 15 & 65.15 & - & 59.84 to 70.46 & $0.12 \dagger(-0.06$ to -0.30$)$ \\
\hline T3 (20 weeks) & 11 & 64.54 & - & 57.65 to 71.43 & 14 & 64.87 & - & 59.60 to 70.14 & $0.03^{*}(-0.14$ to -0.20$)$ \\
\hline \multicolumn{10}{|c|}{ ELDQOL behaviour } \\
\hline T0 (baseline) & 19 & 14.36 & 3.25 & - & 21 & 15.96 & 5.33 & - & - \\
\hline T1 (4 weeks) & 15 & 15.48 & - & 13.92 to 17.03 & 19 & 14.67 & - & 13.29 to 16.06 & $0.26 \dagger(0.07$ to 0.45$)$ \\
\hline T2 (12 weeks) & 15 & 14.39 & - & 12.83 to 15.95 & 16 & 15.25 & - & 13.78 to 16.71 & $0.28^{*}(0.08$ to 0.48$)$ \\
\hline T3 (20 weeks) & 16 & 15.86 & - & 14.33 to 17.39 & 16 & 14.50 & - & 13.03 to 15.96 & $0.43 \dagger(0.21$ to -0.65$)$ \\
\hline \multicolumn{10}{|l|}{ ELDQOL mood } \\
\hline T0 (baseline) & 19 & 28.27 & 6.10 & - & 20 & 30.72 & 7.31 & - & - \\
\hline T1 (4 weeks) & 15 & 31.07 & - & 28.73 to 33.42 & 18 & 29.53 & - & 27.34 to 31.71 & $0.32 \dagger(0.12$ to 0.52$)$ \\
\hline T2 (12 weeks) & 15 & 30.64 & - & 28.27 to 33.00 & 16 & 31.62 & - & 29.32 to 33.93 & $0.21^{*}(0.02$ to 0.40$)$ \\
\hline T3 (20 weeks) & 16 & 31.08 & - & 28.78 to 33.38 & 17 & 30.05 & - & 27.85 to 32.24 & $0.22 \dagger(0.04$ to 0.40$)$ \\
\hline
\end{tabular}

intervention group $(n=21)$ and $10-13$ people in the control condition $(n=19)$.

The fourth feasibility criterion (likelihood of improvement in quality of life) can therefore be considered to be partially met: indication of an effect of the intervention on the two ELDQOL subscales (behaviour and mood), at two out of three time points (T1 and T3), with the most complete data.

Seizure control was a secondary outcome, and was assessed through completion of a seizure diary during the 20-week study period. If participants had not experienced seizures over the previous 4 weeks, then they did not complete the ELDQOL seizure severity subscale at each time point. A sizeable minority of participants reported having no seizures over the previous 4 weeks: a mean of $38 \%$ in the intervention group compared with $25 \%$ in the control group. Similarly, more intervention group participants than control group participants experienced no seizures over the course of the study (33\% compared with 16\%). As would be expected from these results, the intervention group reported fewer seizures over the 20-week study period than did the control group (13.78 (95\% CI -0.36 to 35.61), compared with 17.63 (95\% CI 1.73 to 25.83$)$ ).

\section{Acceptability and feasibility of methodology and} intervention

Interviews with 15 sets of participants and/or carers took place at the 20-week time point and occurred between
January and September 2015. Eleven interviews took place with both participant and carer(s), one participant and their carer were interviewed separately, two interviews took place with the participant only and one interview took place with the carer only. Interviews with four health professionals took place between February and November 2015. The health professionals were all involved in the study, although this varied from close to minimal involvement.

Table 5 shows the themes and subthemes regarding the study methods and intervention from the interviews with participants and carers, and the results indicate that feasibility criteria 5 and 6 were met. Interviewees were generally very positive about their experience of the WIELD project and would recommend participating in the study to others. Some areas for improvement were suggested, for example, offering online questionnaire completion, and reducing the length of the questionnaires. Participants and carers also identified specific questions or subscales, which they found difficult to complete, and which tended to coincide with missing data.

Participants and carers reported that they had received little information about epilepsy in the past, which was accessible for people with learning disabilities. The booklet was generally received very positively by participants and carers, and participants reported relating the booklet to their own experiences. Participants and carers felt that the epilepsy-related conversations 
Table 5 Key themes about acceptability and feasibility from the interviews with participants and carers

\begin{tabular}{|c|c|}
\hline Themes & Subthemes \\
\hline $\begin{array}{l}\text { Feasibility and acceptability of } \\
\text { participation }\end{array}$ & $\begin{array}{l}\text { Most people enjoyed taking part in WIELD and would recommend it to others. } \\
\text { Most people understood the need for random allocation and accepted this was } \\
\text { part of the study. } \\
\text { Study information explained the study well and was suitable for most participants. } \\
\text { Study visit went well and everything was explained clearly. }\end{array}$ \\
\hline $\begin{array}{l}\text { Feasibility and acceptability of data } \\
\text { collection methods }\end{array}$ & $\begin{array}{l}\text { Completing the questionnaires four times was fine for most people. } \\
\text { Most participants were involved in completing the questionnaires. } \\
\text { Most people felt that the questions and language were clear but there was some } \\
\text { room for improvement. } \\
\text { options to complete the questionnaire online, on the phone or at a visit. } \\
\text { Some carers and participants sometimes found it challenging to find the time to } \\
\text { complete the questionnaires. } \\
\text { Some people completed the seizure diary but others did not. } \\
\text { Some people thought the questionnaires were quite long but this was not a } \\
\text { problem for most people. }\end{array}$ \\
\hline Use and acceptability of the booklet & $\begin{array}{l}\text { A minority of carers and participants were already familiar with Beyond Words. } \\
\text { Most participants engaged with the booklet and could identify benefits. } \\
\text { Control participants had differing views on the booklet after first looking at it. } \\
\text { The booklet was used in different ways. }\end{array}$ \\
\hline Feasibility of routine use of the booklet & $\begin{array}{l}\text { Mixed feelings about level of support needed to use the booklet. } \\
\text { Need to be aware if person with learning disabilities is anxious as the booklet } \\
\text { could make this worse. } \\
\text { The booklet could be implemented in the health and social care system. } \\
\text { The booklet could be useful for other people with epilepsy. } \\
\text { The booklet is useful for people with difficulties with verbal communication. } \\
\text { The booklet may be most useful when someone has just been diagnosed. } \\
\text { The time needed to use the booklet could be a barrier. }\end{array}$ \\
\hline
\end{tabular}

WIELD, Wordless Intervention for Epilepsy in Learning Disabilities.

prompted by the booklet were helpful, and revealed new information. Several participants also noted that the booklet helped them to further understand epilepsy and to explain their condition to others. Participants with well-controlled epilepsy felt that the intervention may be more appropriate for people who had recently been diagnosed, or had difficulties understanding or managing their epilepsy. Some carers and participants felt that they needed the guidance provided in the intervention session in order to be able to use the booklet, whereas others felt that they would be able to use the booklet without this.

The interviews with health professionals largely supported the observations made by participants and carers and suggested ways to improve the set-up and recruitment phase of the study. Participating health professionals felt that carers needed support to use the booklet appropriately. There were mixed views about the group of participants who this intervention may be most effective for. The booklet was reported to be more difficult to use with non-verbal participants, although the health professionals acknowledged that the booklet was designed for use with this population and this group often had the most severe epilepsy. It was also suggested that although the booklet had the potential to be useful for all people with learning disabilities and epilepsy, it may be most useful for people who had recently been diagnosed.

In addition to the qualitative data, quantitative data about the use of the booklet during the study were collected. The intervention session took place in one sitting and took $\sim 20 \mathrm{~min}$. The majority of people in the intervention group used the booklet at least twice after the intervention session $(\mathrm{n}=14)$, with three participants never using the booklet at home. The number of people reporting to have used the booklet at home since the previous questionnaire decreased over the course of the study from 15 at $\mathrm{T} 1$, to 12 at T2, and to 10 at T3. The frequency and time spent using the booklet also decreased over time. At T3 those who were still using the booklet reported to be doing so with a wider range of people than at $\mathrm{T} 1$. There were no reported harms or unintended effects in either group.

\section{Health economic analysis}

Across the 21 intervention patients, the mean per participant cost of the intervention was estimated to be $£ 121.56$, where this was composed of training costs 
Table 6 EQ-5D-5L mean scores, SDs and 95\% Cls

\begin{tabular}{|c|c|c|c|c|c|c|c|c|}
\hline & \multicolumn{4}{|c|}{ Control group } & \multicolumn{4}{|c|}{ Intervention group } \\
\hline & $\overline{\mathbf{N}}$ & Mean & SD & 95\% Cls & $\overline{\mathbf{N}}$ & Mean & SD & $95 \% \mathrm{Cls}$ \\
\hline \multicolumn{9}{|l|}{ EQ-5D-5L index } \\
\hline T0 (baseline) & 19 & 0.461 & - & 0.301 to 0.621 & 21 & 0.611 & - & 0.480 to 0.743 \\
\hline T1 (4 weeks) & 15 & 0.494 & - & 0.291 to 0.696 & 18 & 0.643 & - & 0.459 to 0.692 \\
\hline T2 (12 weeks) & 14 & 0.497 & - & 0.263 to 0.731 & 16 & 0.594 & - & 0.436 to 0.752 \\
\hline T3 (20 weeks) & 13 & 0.622 & - & 0.404 to 0.840 & 17 & 0.661 & - & 0.504 to 0.818 \\
\hline QALY & 13 & 0.219 & - & 0.139 to 0.299 & 17 & 0.241 & - & 0.188 to 0.294 \\
\hline \multicolumn{9}{|l|}{ EQ-VAS } \\
\hline T0 (baseline) & 19 & 77.37 & 16.45 & - & 21 & 70.62 & 27.30 & - \\
\hline T1 (4 weeks) & 15 & 71.33 & 21.25 & - & 18 & 76.89 & 23.84 & - \\
\hline T2 (12 weeks) & 15 & 83.67 & 17.78 & - & 16 & 74.88 & 20.17 & - \\
\hline T3 (20 weeks) & 15 & 78.20 & 21.53 & - & 16 & 76.69 & 25.52 & - \\
\hline
\end{tabular}

(£28.42 per participant), an initial meeting cost (£72.14 per participant, based on a 21 min meeting with travel time and costs included), a follow-up phone call (£11.00 per participant) and the cost of the book (£10.00). The seventh feasibility criterion states that a minimal increase in treatment cost should be evident; therefore, we judge this to be partially met. However, we estimate that given the research context, this cost is significantly higher than what it would cost to use the booklet in routine care (eg, to be conservative all training costs were apportioned across the small number of participants in the intervention arm of the study). Paired cost and EQ-5D-5L data were available for 29/40 participants at the 20-week follow-up point (16 intervention, 13 control), demonstrating that it was generally feasible to collect the economic data in the devised manner. All subsequently reported results are based on these 29 participants. Over the 20-week follow-up period, mean NHS costs (excluding the cost of the intervention) were $£ 616.79$ and $£ 698.46$ for the intervention and control arm, respectively. Based on the regression analyses, which adjusted for baseline costs and included the cost of the intervention, over the 20-week follow-up period, mean NHS costs were estimated to be $£ 87.08$ (95\% CI -375.77 to 549.94) higher for the intervention group compared with the control group.

Total mean NHS, PSS and informal care costs (which included residential costs, where applicable) were substantially higher and estimated to amount to $£ 36656.96$ for the intervention and $£ 45430.40$ for the control arm, respectively. The mean cost difference between groups was not estimated to be significant ( $-£ 2663.39,95 \%$ CI -8480.29 to 3151.51$)$. Quality of life as assessed by the standard EQ-5D-5L questionnaire can be seen in table 6 . The mean QALY difference was -0.006 (95\% CI -0.050 to 0.038$)$. The corresponding EQ-5D visual analogue scale (0-100 with higher scale indicating better quality of life) was also similar between treatment groups. It can therefore be seen that there was no significant difference between either the mean costs or QALY score for the intervention group compared with the control group.

\section{DISCUSSION}

\section{Principal findings}

RCTs are the gold standard for evaluating interventions and services. This study is the first completed RCT addressing the outstanding information and selfmanagement needs of people with learning disabilities and epilepsy. Recruitment was extended by 4 months and achieved the target, without delaying the study's end date. Eighty-five per cent of study measures were completed and attrition was minimal (one death unrelated to study involvement and no other drop-out). A potential effect of the intervention on two ELDQOL subscales was found at 4 and 20 weeks. The acceptability and feasibility of the methodology and intervention were high among participants, carers and health professionals. Collection of economic data was found to be feasible. All feasibility criteria were fully met $(n=4)$ or partially met $(n=3)$ and qualitative and quantitative findings indicated improvements to the recruitment and data collection procedures, thus confirming the feasibility of undertaking a definitive trial.

\section{Strengths and weaknesses}

The strengths of this study were the rigorous randomised controlled evaluation of the feasibility of this pictorial intervention. This approach has very rarely been adopted before in the context of learning disabilities research. ${ }^{15}$ Second, there were high levels of public and patient involvement, including people with learning disabilities and their carers, which significantly strengthened the study design, management and dissemination of the study findings. ${ }^{48}$ It is hoped that the successful delivery of this study paves the way for further research in this area with similarly robust methodology, supported by continuous stakeholder involvement.

However, the following limitations need to be considered. First, the proportion of people with an epilepsy diagnosis increases with the severity of learning disabilities, with a recent prevalence estimate of $42 \%$ in people with 'more severe' learning disabilities. ${ }^{5}$ Only four study participants had limited or no verbal communication 
(two in each group), which is not representative of this group of patients. During recruitment, it became clear that many carers of patients with severe learning disabilities and/or non-verbal communication felt that they did not meet the inclusion criteria of being able to interact with or follow the booklet's story, although clinicians had initially identified the patient as eligible during screening $(n=21)$. As the booklet was designed to be used by people with a wide range of learning disabilities and communication abilities, this would need to be addressed in a full-scale trial by ensuring that the initial material presented to carers highlights the study's and intervention's suitability for people with severe learning disabilities and/or limited verbal communication. Second, this study relied on proxy completion of the questionnaires, in order to standardise questionnaires across all participants. From the qualitative data, it is evident that carers involved people with learning disabilities to different degrees and that some participants with mild learning disabilities completed the questionnaires independently. There is evidence to suggest that there are differences in the responses of patients, family carers and paid carers on questionnaires of epilepsy-related concerns. ${ }^{50}{ }^{51}$ In future work, the variation across different respondents could be examined.

\section{Research in context}

People with learning disabilities are under-represented in clinical trials, which results in a limited knowledge base about effective healthcare interventions in a population already experiencing health disparities. ${ }^{12} 19$ There are modifications and adaptations that can be made to study designs and procedures to minimise obstacles including accessible information materials (eg, easy-read formats were successfully used in WIELD-see online supplementary material), simplified consent procedures, modified procedures to allow support from carers and simplified data collection materials. ${ }^{19}$ People with learning disabilities are positive about participating in research and highlight the importance of researchers understanding their experiences and opinions. ${ }^{52}$

Two key factors in this study facilitated successful recruitment and high levels of acceptability of the design. First, the study procedures and materials were carefully designed with the feedback of people with learning disabilities, carers, members of the public and health professionals, which resulted in improved accessibility. Second, the design of this study was closely aligned with local epilepsy and learning disability services, which facilitated recruitment. It is important for clinical trials to be embedded within the relevant health services to ensure successful recruitment and likelihood of potential implementation. ${ }^{53}{ }^{55}$ Services across England for people with learning disabilities and epilepsy vary greatly; ${ }^{15}$ the clinical care pathway in different NHS Trusts may affect recruitment in a full-scale trial and routes to implementation in routine care. Therefore, future research and dissemination would need to continue to work collaboratively with local services, and explore different ways of using the booklet and how this may, or may not, align with local practice.

A recent scoping review of self-management interventions for people with learning disabilities and epilepsy ${ }^{25}$ identified a nurse-led intervention that is currently underway, ${ }^{55}$ and three completed small-scale studies. ${ }^{56-58}$ The results of the completed studies are promising and suggest that a self-management intervention could be appropriate and beneficial for people with learning disabilities and epilepsy. However they are underpowered, did not use a RCT design and did not assess outcomes on quality of life or seizure control.

\section{Implications for clinicians and policymakers}

Very few of the study participants had ever received information about epilepsy that was adapted to their communication ability. Much of the information provided by health professionals was not easily accessible, and was aimed at carers. The booklet was received positively and various benefits were noted. This suggests that there is a need for tailored education and selfmanagement information for people with learning disabilities and epilepsy, which is currently unmet. The feasibility findings are promising, but the results of a definitive trial are necessary to demonstrate whether the proposed intervention meets these needs, and improves outcomes.

The qualitative data suggested that some participants experienced improvements from the intervention in their epilepsy-related knowledge, confidence and anxiety. There is currently no measure that assesses these constructs in people with learning disabilities and epilepsy. Further research could explore this to understand more about the needs of this population, and how to meet these needs.

This study did not explore the fidelity of the intervention; in order to fully evaluate this intervention, it would be crucial to ascertain whether the intervention is used by participants and carers as recommended, and to explore and understand the underlying mechanisms of booklet use. A process evaluation approach using ethnographic methods would be informative to observe how the booklet is used, and what the 'critical ingredients' of this intervention may be.

\section{CONCLUSIONS}

The WIELD study was completed with successful recruitment and minimal missing data. Four out of seven feasibility criteria were fully met, and three criteria were partially met, thus confirming the feasibility of undertaking a definitive trial. Acceptability of the study methods and intervention was high among participants, carers and health professionals. By developing and testing methods to reduce the number of follow-ups, extend the recruitment period, develop study information material to increase the involvement of participants with severe learning disabilities and assess epilepsy knowledge, 
recruitment rates could increase and the effects of the intervention could be measured more accurately. Given the success of the feasibility study and robust evidence to address any potential issues, it is clear that a definitive trial is both feasible and necessary to address important gaps in evidence-based practice for people with learning disabilities and epilepsy.

\section{Author affiliations}

${ }^{1}$ Department of Psychology, Centre for Health Services and Clinical Research, University of Hertfordshire, Hatfield, UK

${ }^{2}$ Institute for Practice, Interdisciplinary Research and Enterprise (INSPIRE), University of West London, London, UK

${ }^{3}$ Learning Disabilities Services, Hertfordshire Partnership University NHS Foundation Trust, St Albans, UK

${ }^{4}$ Norwich Medical School and Norwich Clinical Trials Unit, University of East Anglia, Norwich, UK

${ }^{5}$ Department of Psychiatry, University of Cambridge, School of Clinical Medicine, Cambridge, UK

${ }^{6}$ Research and Development, Hertfordshire Partnership University NHS Foundation Trust, St Albans, UK

${ }^{7}$ Division 4, Mental Health, NIHR Clinical Research Network: Eastern, UK ${ }^{8}$ Centre for Research in Primary and Community Care, University of Hertfordshire, Hatfield, UK

${ }^{9}$ The Dartmouth Institute for Health Policy and Clinical Practice, Dartmouth College, Hanover, New Hampshire, USA

Acknowledgements The study team gratefully acknowledge Alan Lim, Vassen Karuthasami and the Consultant Psychiatrists who assisted with recruitment. The following are also thanked for their continued support and valuable contributions: Patient Involvement in Research Group at the University of Hertfordshire, The Making Better Services Group, British Institute of Learning Disabilities, the East of England Research Design Service, The Norwich Clinical Trials Unit, Beyond Words, and Professor Tim Gale at Hertfordshire Partnership University NHS Foundation Trust.

Contributors M-AD, BG, GP, AZ, KF, GB, HR and DW participated in the design and conception of the study. MEK, DM-P, SEM, GP and AZ recruited participants. SEM, MEK and DM-P collected data. SEM, DW, M-AD, LI and GB analysed data. SEM drafted the article, which was revised by M-AD. All authors commented on all subsequent drafts. All authors read and approved the final version of the manuscript.

Funding This manuscript presents independent research funded by the National Institute for Health Research (NIHR) under its Research for Patient Benefit (RfPB) Programme (grant reference number PB-PG-0213-30042).

Competing interests None declared.

Ethics approval This study was approved by NRES Wales Research Ethics Committee 5 in April 2014 (Ref: 14/WA/0135).

Provenance and peer review Not commissioned; externally peer reviewed.

Data sharing statement All requests should be directed to the corresponding author.

Open Access This is an Open Access article distributed in accordance with the terms of the Creative Commons Attribution (CC BY 4.0) license, which permits others to distribute, remix, adapt and build upon this work, for commercial use, provided the original work is properly cited. See: http:// creativecommons.org/licenses/by/4.0/

\section{REFERENCES}

1. Krahn GL, Hammond L, Turner A. A cascade of disparities: health and health care access for people with intellectual disabilities. Ment Retard Dev Disabil Res Rev 2006;12:70-82.

2. van Schrojenstein Lantman-de Valk HM, Walsh PN. Managing health problems in people with intellectual disabilities. $B M$ 2008;337:a2507.
3. Heslop $\mathrm{P}$, Blair $\mathrm{P}$, Fleming $\mathrm{P}$, et al. Confidential inquiry into premature deaths of people with learning disabilities (CIPOLD) 2013. http://www.bris.ac.uk/cipold/reports/

4. van Schrojenstein Lantman-De Valk HM, Metsemakers JF, Haveman $\mathrm{MJ}$, et al. Health problems in people with intellectual disability in general practice: a comparative study. Fam Pract 2000;17:405-7.

5. Robertson J, Hatton C, Emerson E, et al. Prevalence of epilepsy among people with intellectual disabilities: a systematic review. Seizure 2015;29:46-62.

6. World Health Organisation. Epilepsy: Fact Sheet No. 999. 2015. http://www.who.int/mediacentre/factsheets/fs999/en/

7. Matthews $\mathrm{T}$, Weston $\mathrm{N}$, Baxter $\mathrm{H}$, et al. A general practice-based prevalence study of epilepsy among adults with intellectual disabilities and of its association with psychiatric disorder, behaviour disturbance and carer stress. J Intellect Disabil Res 2008;52:163-73.

8. McGrother CW, Bhaumik S, Thorp CF, et al. Epilepsy in adults with intellectual disabilities: prevalence, associations and service implications. Seizure 2006;15:376-86.

9. Kerr M, Scheepers M, Arvio M, et al. Consensus guidelines into the management of epilepsy in adults with an intellectual disability. $J$ Intellect Disabil Res 2009;53:687-94.

10. McCarron M, O'Dwyer M, Burke E, et al. Epidemiology of epilepsy in older adults with an intellectual disability in Ireland: associations and service implications. Am J Intellect Dev Disabil 2014;119:253-60.

11. Glover G, Evison F. Hospital admissions that should not happen: improving health and lives. Learning Disabilities Observatory. 2013. https://www.improvinghealthandlives.org.uk publications $/ 1169$

12. Kerr M, Linehan C, Thompson R, et al. A White Paper on the medical and social needs of people with epilepsy and intellectual disability: the Task Force on Intellectual Disabilities and Epilepsy of the International League Against Epilepsy. Epilepsia 2014;55:1902-6.

13. Bowley C, Kerr M. Epilepsy and intellectual disability. J Intellect Disabil Res 2000;44:529-43.

14. Robertson J, Hatton C, Emerson E, et al. Mortality in people with intellectual disabilities and epilepsy: a systematic review. Seizure 2015;29:123-33.

15. Robertson J, Baines S, Emerson E, et al. Service responses to people with intellectual disabilities and epilepsy: a systematic review. J Appl Res Intellect Disabil 2015.

16. National Institute for Health and Care Excellence. The epilepsies: the diagnosis and management of the epilepsies in adults and children in primary and secondary care. NICE clinical guideline 137: NICE, 2012. http://www.nice.org.uk/guidance/cg137

17. Reuber M, Gore J, Wolstenhome J, et al. Examining a community model of epilepsy care for people with learning disabilities. Seizure 2008;17:84-91.

18. van Blarikom W, Tan IY, Aldenkamp AP, et al. Epilepsy, intellectua disability, and living environment: a critical review. Epilepsy Behav 2006:9:14-18.

19. Feldman MA, Bosett $\mathrm{J}$, Collet $\mathrm{C}$, et al. Where are persons with intellectual disabilities in medical research? A survey of published clinical trials. J Intellect Disabil Res 2014:58:800-9.

20. Hannah JA, Brodie MJ. Epilepsy and learning disabilities-a challenge for the next millennium? Seizure 1998;7:3-13.

21. Beavis J, Kerr M, Marson AG. Non-pharmacological interventions for epilepsy in people with intellectual disabilities. Cochrane Database Syst Rev 2007:4:CD005502.

22. Bradley PM, Lindsay B. Care delivery and self-management strategies for adults with epilepsy. Cochrane Database Syst Rev 2008;(1):CD006244.

23. Ramaratnam S, Baker GA, Goldstein LH. Psychological treatments for epilepsy. Cochrane Database Syst Rev 2008;(3):CD002029.

24. Edward KL, Cook M, Giandinoto J-A. An integrative review of the benefits of self-management interventions for adults with epilepsy. Epilepsy Behav 2015;45:195-204.

25. Dannenberg M, Mengoni SE, Gates B, et al. Self-management interventions for epilepsy in people with intellectual disabilities: a scoping review. Seizure 2016;41:16-25.

26. Hollins S, Bernal J, Thacker A, et al. Getting on with epilepsy. 2nd edn. UK: St George's Hospital Medical School and Gaskell, 2014.

27. Beyond Words. What are Books Beyond Words n.d. http:// booksbeyondwords.co.uk/about/

28. Cherry KE, Applegate H, Reese CM. Do adults with mental retardation show pictorial superiority effects in recall and recognition? Res Dev Disabil 2002;23:135-47.

29. Houts PS, Doak CC, Doak LG, et al. The role of pictures in improving health communication: a review of research on attention, 
comprehension, recall, and adherence. Patient Educ Couns 2006;61:173-90.

30. Alberto PA, Fredrick L, Hughes M, et al. Components of visual literacy: teaching logos. Focus Autism Other Dev Disabl 2007;22:234-43.

31. Durand MA, Gates B, Parkes G, et al. Wordless intervention for epilepsy in learning disabilities (WIELD): study protocol for a randomized controlled feasibility trial. Trials 2014;15:455

32. Buck D, Smith M, Appleton R, et al. The development and validation of the Epilepsy and Learning Disabilities Quality of Life (ELDQOL) scale. Epilepsy Behav 2007;10:38-43.

33. Baker GA, Jacoby A, Smith DF, et al. Development of a novel scale to assess life fulfillment as part of the further refinement of a quality-of-life model for epilepsy. Epilepsia 1994;35:591-6.

34. Herdman M, Gudex C, Lloyd A, et al. Development and preliminary testing of the new five-level version of EQ-5D (EQ-5D-5L). Qual Life Res 2011;20:1727-36.

35. Mengoni SE, Gates B, Parkes G, et al. "Sometimes, it just stops me from doing anything": a qualitative exploration of epilepsy management in people with intellectual disabilities and their carers. Epilepsy Behav 2016;64(Pt A):133-9.

36. Cocks K, Torgerson DJ. Sample size calculations for pilot randomized trials: a confidence interval approach. J Clin Epidemiol 2013;66:197-201.

37. Department of Health. National Schedule of Reference Costs 201314. 2014. https://www.gov.uk/government/publications/nhs-referencecosts-2013-to-2014

38. Curtis L. Unit costs of health and social care. The University of Kent 2014.

39. NHS: The Information Centre for health and social care. Prescription Cost Analysis: England 2013. Health and social care information centre, prescribing and primary care services: health and social care information centre, 2014.

40. Office for National Statistics. Annual Survey of Hours and Earnings. 2013. http://www.ons.gov.uk/ons/rel/ashe/annual-survey-of-hoursand-earnings/2013-revised-results/index.html

41. Drummond MF, Sculpher MJ, Claxton K, et al. Methods for the economic evaluation of health care programmes. 4th edn. Oxford: Oxford University Press, 2015.

42. van Hout $B$, Janssen MF, Feng $Y S$, et al. Interim scoring for the EQ-5D-5L: mapping the EQ-5D-5L to EQ-5D-3L value sets. Value Health 2012;15:708-15.

43. Manca A, Hawkins N, Sculpher MJ. Estimating mean QALYs in trial-based cost-effectiveness analysis: the importance of controlling for baseline utility. Health Econ 2005;14:487-96.
44. Willan AR, Briggs $A H$, Hoch JS. Regression methods for covariate adjustment and subgroup analysis for non-censored cost-effectiveness data. Health Econ 2004;14:461-75.

45. Noble SM, Hollingworth W, Tilling K. Missing data in trial-based cost-effectiveness analysis: the current state of play. Health Econ 2012;21:187-200.

46. NICE. Guide to the methods of technology appraisal 2013 National Institute of Health and Clinical Excellence (NICE) publications, 2013

47. Braun V, Clarke V. Using thematic analysis in psychology. Qual Res Psychol 2006;3:77-101.

48. Mengoni S, Gates B, Durand MA. An intervention for people with learning disabilities and epilepsy. Learn Disabil Pract 2015;49:28-31.

49. Bigby C, Frawley P, Ramcharan P. Conceptualizing inclusive research with people with intellectual disability. J Appl Res Intellect Disabil 2014;27:3-12.

50. Watkins J, Espie CA, Curtice L, et al. Development of a measure to assess the impact of epilepsy on people with an intellectual disability: the Glasgow Epilepsy Outcome Scale-Client version (GEOS-C). J Intellect Disabil Res 2006;50:161-71.

51. Espie CA, Watkins J, Duncan R, et al. Perspectives on epilepsy in people with intellectual disabilities: comparison of family carer, staff carer and clinician score profiles on the Glasgow Epilepsy Outcome Scale (GEOS). Seizure 2003;12:195-202.

52. McDonald KE, Kidney CA, Patka M. 'You need to let your voice be heard': research participants' views on research. $J$ Intellect Disabil Res 2013;57:216-25

53. Murray E, Treweek S, Pope C, et al. Normalisation process theory: a framework for developing, evaluating and implementing complex interventions. BMC Med 2010;8:1-11.

54. Craig P, Dieppe $\mathrm{P}$, Macintyre $\mathrm{S}$, et al. Developing and evaluating complex interventions: the new Medical Research Council guidance. BMJ 2008;337:a1655

55. Ring $\mathrm{H}$, Irvine $\mathrm{F}$, Donaldson $\mathrm{C}$, et al. Clinical trial protocolimproving outcomes in adults with epilepsy and intellectual disability: a cluster randomised controlled trial of nurse-led epilepsy management (EpAID). 2014. http://www.nets.nihr.ac.uk/ data/ assets/pdf_file/0014/81131/PRO-10-104-16.pdf

56. Codling M. The ME (My epilepsy) Project: an active learning approach for people with learning disabilities and epilepsy. Epilepsy Care 2010;2:3-7.

57. Clark AJ, Espie CA, Paul A. Adults with learning disabilities and epilepsy: knowledge about epilepsy before and after an educational package. Seizure 2001;10:492-9.

58. Kushinga K. The PEPE project. Learn Disabil Pract 2007;10:10-13. 